\title{
Advanced implementations of phase sensitive amplifiers
}

\author{
F. Parmigiani*, G. Hesketh, K. R. H. Bottrill, R. Slavík, P. Horak, P. Petropoulos, D. J. Richardson \\ Optoelectronics Research Centre, University of Southampton, \\ Southampton, SO17 1BJ, UK \\ frp@orc.soton.ac.uk
}

\begin{abstract}
We review our recent results on different configurations of phase sensitive amplifiers that achieve alloptical phase regeneration and optical phase quantization at low nonlinear phase shifts.
\end{abstract}

Keywords- Nonlinear optics, parametric processes, regeneration.

\section{INTRODUCTION}

In an effort to address the continued growth of data traffic in optical networks, spectrally efficient modulation formats, utilizing both phase and amplitude, are being investigated [1]. The performance of these complex modulation formats can be severely degraded due to the linear phase noise (PN) added by optical amplifiers and the nonlinear PN caused by the fiber nonlinearity [2]. Optical methods that can be used to process these signals, such as optical phase regenerators for M-ary phase shift keying (M-PSK) signals, which potentially offer energy and cost reductions over electronics, format flexibility and ultra-fast processing rates, have been gaining momentum as a key technology to develop.

Phase sensitive amplifiers (PSAs) based on fiber optic parametric amplifiers (FOPA) may be used to achieve some of these optical systems in single- or dual-pump configurations with the signal, idler and $\operatorname{pump}(\mathrm{s})$ being either along the same linear polarization ('scalar schemes') or on different polarization axes ('vector schemes') - and include all possibilities covering both non-degenerate and degenerate (signal and idler at the same wavelength) configurations [3][5]. For example, binary phase shift keying (BPSK) phase (and amplitude) regeneration has been successfully achieved using a scalar dual-pump degenerate PSA in highly nonlinear fibers (HNLFs) [3]. In more detail, the binary step-like phase response is the result of the coherent addition of the original signal and its first four-wave mixing (FWM)-generated complex-conjugate copy (idler) with ideally comparable strength. The scheme can be extended to allow phase regeneration of an arbitrary M-PSK signal format; this can be implemented through a coherent addition of the (M-1)-th phase harmonic of the signal to the signal itself with a mixing factor that depends on the corresponding signal phase levels [4]. Different nonlinear materials can also be considered to achieve PSA and very recently dual-polarization 16-quadrature amplitude modulation (16-QAM) signals carried by three

F. P. gratefully acknowledges the support from the Royal Academy of Engineering/EPSRC through a University research Fellowship. wavelength-division-multiplexed (WDM) channels have been successfully processed in a "copier-PSA" configuration with periodically poled lithium niobate (PPLN) waveguides [6].

In the following, we will focus on two specific configurations of HNLF-based PSAs to achieve: i) optical phase regeneration with negligible phase-to-amplitude conversion as proposed in [7] and demonstrated in [8], and ii) two-level phase quantization with low nonlinear phase shifts (NPSs) [9].

\section{DUAL HARMONIC PSA SCHEME}
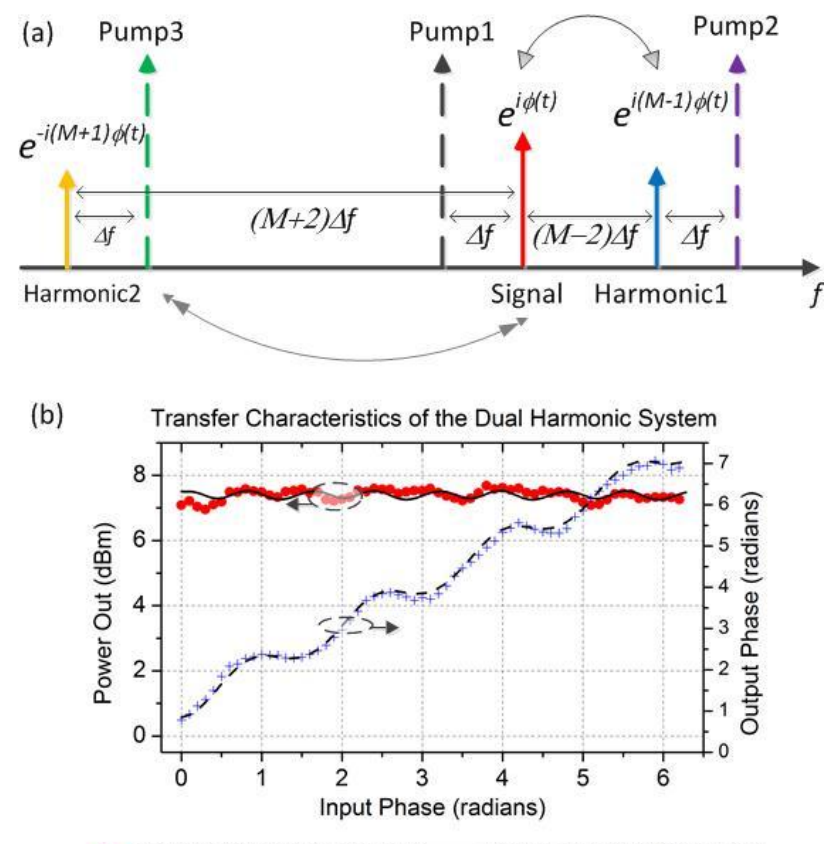

- Power Transfer Measured; - Power Transfer Simulated Phase Transfer Measured; - - - Phase Transfer Simulated

Fig.1 Dual-harmonic PSA scheme operation principle (a) and an example of corresponding measured (red circles and blue crosses) and simulated (solid and dashed lines) signal transfer functions (b). Pump powers were set to $15.3 \mathrm{dBm}$ and the signal power to $7.3 \mathrm{dBm}$ at the input of the HNLF, resulting in a weight of the phase harmonic of about 0.14 as compared to the original signal.

To achieve good phase quantization (regeneration) of $\mathrm{M}$ PSK signals, while greatly reducing the phase-to-amplitude dependence, the scheme proposed in [4], which we refer to as a single harmonic scheme, can be altered by mixing not only the 
(M-1)-th phase harmonic, but also the -(M+1)-th phase harmonic with the original signal [7], as shown in Fig.1 (a). We refer to this scheme as a dual harmonic scheme. Initial experimental results applied to the 4-level phase quantization (appropriate for a QPSK signal) showed similar performance in terms of phase quantization to the conventional singleharmonic system, whilst achieving a reduction in phase dependent gain variation of about $6.5 \mathrm{~dB}$, obtaining an overall amplitude-to-phase variation of less than $0.5 \mathrm{~dB}$ [8]. Fig.1 (b) reports an example of the corresponding simulated and measured amplitude and phase transfer functions.

\section{POLARIZATION-ASSISTED PSA}

A two-level phase (quantized) response (appropriate for phase regeneration of a BPSK signal or demultiplexing of higher complex modulation formats [10]) is the result of the coherent addition of signal and idler (at the same wavelength for the degenerate configuration) with similar weights. For this scenario, the amount of output phase squeezing is proportional to the phase sensitive extinction ratio, PSER, defined as the difference between the maximum phase sensitive (PS) gain and the maximum PS de-amplification. Large symmetric PSERs (i.e. exhibiting an equal maximum PS gain and maximum PS de-amplification) can only be achieved at high pump powers implying the requirements of sophisticated techniques to be employed for the suppression of stimulated Brillouin scattering and resulting in the generation of several FWM components across a wide spectral bandwidth. Such FWM components may in fact enhance the PS deamplification process and provide large (asymmetric) PSER at carefully selected power levels. For example, PSERs of $25 \mathrm{~dB}$ have been experimentally demonstrated in the scalar degenerate dual-pump PSA for a NPS of $0.8 \mathrm{rad}$ [11]. Recently [9], we have demonstrated that high PSER and, thus, binary step-like phase response at significantly lower NPSs can be achieved using a polarizer at the output of a degenerate parametric amplifier with two orthogonally aligned pumps. As shown in Fig.2 (a), by generating an idler in the orthogonal polarization state as compared to the signal and properly choosing the angle of the polarizer at the output (which depends on the strength of the generated idler), it is possible to exactly match the relative strengths of the signal and idler beams along the polarizer's transmission axis even in the instance that the generated idler is significantly weaker than the signal, i.e. at low pump powers. We refer to this scheme as polarization-assisted PSA. Asymmetric PSERs as large as $26 \mathrm{~dB}$ (mainly limited by the polarization extinction of the polarization maintaining components used) for a NPS as low as 0.3 rad were experimentally demonstrated [9], achieving excellent phase squeezing response, as shown in Fig.2. It is worth noting, though, that at low NPS, high PSERs only correspond to different degrees of signal de- amplification rather than net amplification.

\section{CONCLUSION}

We have reviewed our recent results on different configurations of phase sensitive amplifiers to achieve (i) alloptical phase regeneration with minimal phase-to-amplitude
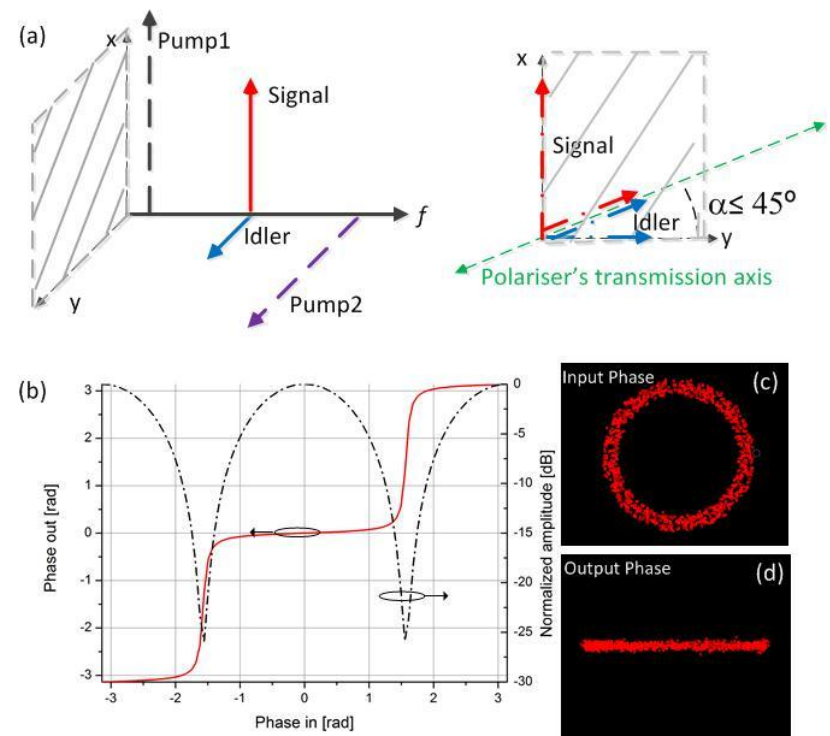

Fig.2 Polarization-assisted PSA scheme operation principle (a) and a typical (simulated) amplitude and phase response versus input phase for a PSER of $26 \mathrm{~dB}$ (b) and corresponding measured constellation diagrams at the input (c) and output (d) of the system for the same PSER achieved at a NPS of 0.3 rad. The initial signal was passed through a phase modulator to induce continuous phase variations of over $2 \pi$.

noise conversion using a dual-harmonic scheme and (ii) a binary step-like phase response at low nonlinear phase shifts using a polarization-assisted PSA.

\section{REFERENCES}

[1] M. Y. Frankel and J. Livas, 'High speed optical networks', in Optical Transmission, Switching, and Subsystem II, Pts 1 and 2, vol. 5625, C. F. Lam, W. Y. Gu, N. Hanik, and K. Oguchi, Eds. Bellingham: SpieInt Soc Optical Engineering, 2005, pp. 374-385.

[2] J. P. Gordon and L. F. Mollenauer, "Phase noise in photonic communication systems using linear amplifiers," Opt. Lett., vol. 15, no. 23, pp. 1351-1354, Dec. 1990.

[3] R. Slavk, et al., "All-optical phase and amplitude regenerator for nextgeneration telecommunications systems," Nature Photonics, vol. 4, no. 10, pp. 690-695, Oct. 2010.

[4] J. Kakande, et al., "Multilevel quantization of optical phase in a novel coherent parametric mixer architecture," Nature Photonics, vol. 5, no. 12, pp. 748-752, Dec. 2011.

[5] Z. Tong, et al., "Towards ultrasensitive optical links enabled by lownoise phase-sensitive amplifiers", Nature Photonics, vol. 5, pp. 430436, 2011.

[6] T. Umeki, et al., "First demonstration of high-order QAM signal amplification in PPLN-based phase sensitive amplifier", Optics Express, vol. 22 ( 3), pp. 2473-2482, 2014.

[7] G. Hesketh, P. Horak, "Reducing bit-error rate with optical phase regeneration in multilevel modulation formats", Opt. Lett., 38 53575360 (2013).

[8] K. R. H. Bottrill, et al., "An Optical Phase Quantiser Exhibiting Suppressed Phase Dependent Gain Variation", OFC, W3F.7, 2014.

[9] F. Parmigiani, et al. "Novel Polarization-assisted Phase Sensitive Optical Signal Processor Requiring Low Nonlinear Phase Shifts" submitted to CLEO US 2014.

[10] M. Gao et al., "Low-penalty Phase De-multiplexing of QPSK Signal by Dual-pump Phase Sensitive Amplifiers", ECOC, We.3.A.5 (2013).

[11] M. Gao, T. Kurosu, T. Inoue and S. Namiki, "Efficient phase regeneration of DPSK signal by sideband-assisted dual-pump phase sensitive amplifier", Electronics Lett., 39 (2), 140-141 (2013). 